\title{
THE PRIVATE OWNERSHIP ECONOMY WITH A REDUCED CONSUMPTION SPHERE AS AN ECONOMIC MECHANISM
}

\author{
Agnieszka Lipieta
}

\begin{abstract}
Let $E_{p}$ be a Debreu private ownership economy in which every consumption set is contained in the proper subspace $V$ of the commodity - price space $\mathbb{R}^{\ell}$. Two kinds of economic mechanisms, in the sense of Hurwicz, are designed. The goals of the design are, either the modified to the subspace $V$, producers' and consumers' optimal plans (plans maximizing appropriately profits or preferences on the budget sets) which form the state of equilibrium, or the modified to the subspace $V$ production sets. In the presented economic mechanisms, the producers' maximal profits do not decrease, and also the consumers' preferences and the budget sets remain unchanged.
\end{abstract}

Keywords: Debreu economy, complementary commodities, projections, equilibrium.

JEL Classification: D50, C02.

DOI: $10.15611 / \mathrm{me} .2013 .9 .04$.

\section{Introduction}

This paper is the continuation of the research elaborated in (Lipieta 2010). We are trying to analyze the evolution of a Debreu private ownership economy, in which all consumption sets are contained in a proper subspace $V$ of the commodity - price space $\mathbb{R}^{\ell}, \ell \in\{1,2, \ldots\}$. Producers, in the spirit of competitive mechanism, want to maximize their profits by making consumers better off. Hence, they adjust the quantities of commodities in their production plans to the given relationship. Such a modification of the production sphere leads to an economy in which production and consumption sets are contained in the subspace $V$ (see (Lipieta 2010)). The structure of the above mentioned modifications suggests that the final economy reduced

\footnotetext{
Agniszka Lipieta

Department of Mathematics, Cracow University of Economics, Rakowicka Street 27,

31-510 Kraków, Poland.

E-mail: agnieszka.lipieta@uek.krakow.pl
} 
to the subspace $V$, as well as the process of establishing an equilibrium in the modified economy, are economic mechanisms in the sense of Hurwicz's theory of economic mechanisms.

The paper consists of five parts. In the next section, the definition and the structure of action of the Debreu private ownership economy is presented. The third part contains the basic definitions on the theory of economic mechanisms, the fourth part gives a short description of subspaces in space $\mathbb{R}^{\ell}$. The fifth part contains examples of designing economic mechanisms realizing the given goal correspondence in which the goals are plans contained in the subspace $V$.

\section{Model}

The private ownership Debreu economy, introduced in (Debreu 1959), is defined in the form of a relational system consisting of a combination of production and consumption systems (see for instance (Malawski 2005), (Lipieta 2010). Let $\ell, n \in\{1,2, \ldots\}$. Then

- the linear space $\mathbb{R}^{\ell}$ with the scalar product

$$
(x \circ y)=\left(x_{1}, \ldots, x_{\ell}\right) \circ\left(y_{1}, \ldots, y_{\ell}\right)=\sum_{k=1}^{\ell} x_{k} \cdot y_{k},
$$

is interpreted as the $\ell$ - dimensional commodity - price space,

- $J=\{1, \ldots, n\}$ is a finite set of producers,

- $y: J \ni j \rightarrow Y^{j} \subset \mathbb{R}^{\ell}$ is the correspondence of production sets, which to every producer $j$ assigns a production set $Y^{j} \subset \mathbb{R}^{\ell}$ of the producer's feasible production plans,

- $p \in \mathbb{R}^{\ell}$ denotes a price vector.

The following definitions may be assumed on the basis of the above:

Definition 2.1. If for the given price vector $p \in \mathbb{R}^{\ell}$

$$
\forall j \in J \eta^{j}(p) \stackrel{\text { def }}{=}\left\{y^{j^{*}} \in Y^{j}: p \circ y^{j^{*}}=\max \left\{p \circ y^{j}: y^{j} \in Y^{j}\right\}\right\} \neq \varnothing
$$

then

- $\eta: J \ni j \rightarrow \eta^{j}(p) \subset \mathbb{R}^{\ell}$ is called the correspondence of supply, which to every producer $j \in J$ assigns the set $\eta^{j}(p)$ of production plans maximizing their profit at given price system $p$; the plans belonging to set $\eta^{j}(p)$ will be called the optimal plans of producer $j$, 
- $\pi: J \ni j \rightarrow \pi^{j}(p) \in \mathbb{R}$ is the maximal profit function at given price system $p$, where for every $j \in J, \pi^{j}(p)=p \circ y^{j^{*}}$ for $y^{j^{*}} \in \eta^{j}(p)$,

- the two - range relational system $P=\left(J, \mathbb{R}^{\ell} ; y, p, \eta, \pi\right)$ is called the production system.

Similarly, let $m \in\{1,2, \ldots\}$. Then

- $I=\{1, \ldots, m\}$ is a finite set of consumers,

- $\Xi \subset \mathbb{R}^{\ell} \times \mathbb{R}^{\ell}$ is the family of all preference relations defined on commodity space $\mathbb{R}^{\ell}$

- $\chi: I \ni i \rightarrow X^{i} \subset \mathbb{R}^{\ell}$ is the correspondence of consumptions sets which to every consumer $i \in I$ assigns a consumption set $\chi(i)=X^{i}$ being a subset of the commodity space and representing the consumer's feasible consumption plans,

- $e: I \ni i \rightarrow e^{i} \in X^{i}$ is the initial endowment mapping which to every consumer $i \in I$ assigns the initial endowment vector $e^{i} \in X^{i}$,

- $\varepsilon \subset I \times\left(\mathbb{R}^{\ell} \times \mathbb{R}^{\ell}\right)$ is the correspondence of preference relations, which to every consumer $i \in I$ assigns a preference relation $\preceq^{i}$ from the set $\Xi$ restricted to the consumption set $X^{i}$,

- $p \in \mathbb{R}^{\ell}-$ is a price vector.

Notice that the expenditure of every consumer $i \in I$ is not greater than the value

$$
w^{i}=p \circ e^{i}
$$

Now we can assume the following definition:

Definition 2.2. If for the given price vector $p \in \mathbb{R}^{\ell}$ and for every $i \in I$,

$$
\begin{gathered}
\beta^{i}\left(p, w^{i}\right) \stackrel{\text { def }}{=}\left\{x \in \chi(i): p \circ x \leq w^{i}\right\} \neq \varnothing \\
\varphi^{i}\left(p, w^{i}\right) \stackrel{\text { def }}{=}\left\{x^{i^{*}} \in \beta^{i}\left(p, w^{i}\right): \forall x^{i} \in \beta^{i}\left(p, w^{i}\right) x^{i} \preceq^{i} x^{i^{*}}, \preceq^{i} \in \Xi\right\} \neq \varnothing
\end{gathered}
$$

then 
- $\beta: I \ni i \rightarrow \beta^{i}\left(p, w^{i}\right) \subset \mathbb{R}^{\ell}$ is the correspondence of budget sets, which to every consumer assigns his set of budget constraints $\beta^{i}(p) \subset \chi(i)$ at the price system $p$ and the initial endowment $e(i)$,

- $\varphi: I \ni i \rightarrow \varphi^{i}\left(p, w^{i}\right) \subset \mathbb{R}^{\ell}$ is the demand correspondence at given price system $p$, which to every consumer $i \in I$ assigns his consumption plans maximizing his preference on the budget set $\beta^{i}\left(p, w^{i}\right)$; the plans belonging to set $\varphi^{i}\left(p, w^{i}\right)$ will be called the optimal plans of consumer $i$,

- the three - range relational system $C=\left(I, \mathbb{R}^{\ell}, \Xi ; \chi, e, \varepsilon, p, \beta, \varphi\right)$ is called the consumption system.

Let $p \in \mathbb{R}^{\ell}$ be a price vector, $P$ - a production system and $C$ - a consumption system in the same space $\mathbb{R}^{\ell}$. Moreover, it is assumed that every consumer shares in the producers' profits.

Definition 2.3. The relational system $E_{p}=(P, C, \theta, \omega)$, where

- $P$ is the production system,

- the mapping

$$
\theta: I \times J \rightarrow[0,1], \quad \text { satisfying } \forall j \in J \sum_{i=1}^{m} \theta(i, j)=1
$$

is given; the number $\theta(i, j)$ indicates that part of the profit of producer $j$ which is owned by consumer $i$,

- $C$ is the consumption system in which

$$
\begin{gathered}
w^{i}=p \circ \omega^{i}+\sum_{j=1}^{n} \theta(i, j) \cdot \pi(j), \\
\omega=\sum_{i=1}^{m} e(i) \in \mathbb{R}^{\ell},
\end{gathered}
$$

is called the private ownership Debreu economy (in brief: the Debreu economy).

The vector $\omega$, by (2.7), is called the total endowment of economy $E_{p}$. It is well known that a Debreu economy $E_{p}$ operates as follows. Let a price 
vector $p \in \mathbb{R}^{\ell}$ be given. Every producer $j$ chooses a production optimal plan $y^{j^{*}} \in \eta^{j}(p) \subset Y^{j}$ maximizing his profit at the price system $p$. The maximal profit of each producer is divided among all the consumers according to function $\theta$ (see (2.5)). Every consumer $i$ chooses his optimal consumption plan $x^{i *} \in \varphi^{i}(p) \subset X^{i}$ maximizing his preference on the budget set $\beta^{i}\left(p, w^{i}\right)$, where $w^{i}$ is given by (2.6). If

$$
\sum_{i=1}^{m} x^{i^{*}}-\sum_{j=1}^{n} y^{j^{*}}=\omega
$$

which means the total supply equals the total demand (so the Walras Law is satisfied), then vector $p$ is called the equilibrium price vector and it is denoted by $p^{*}$. The sequence

$$
\left(x^{1^{*}}, \ldots, x^{m^{*}}, y^{1^{*}}, \ldots, y^{n^{*}}, p^{*}\right) \in\left(\mathbb{R}^{\ell}\right)^{m+n+1}
$$

is called the state of Walras equilibrium in the private ownership economy $E_{p}$ (Malawski 2005).

\section{The economic mechanisms}

Now we present some aspects of the theory of designing economic mechanisms which will be of use later. The definitions are borrowed from (Arrow, Intriligator 1987), (Hurwicz, Reiter 2006) and (Werner 2010). It is assumed that $r(r=1,2, \ldots)$ economic agents operate on the market. The set of agents is denoted by $A=\left\{a_{1}, \ldots, a_{r}\right\}$. The set $E$ of characteristics of all economic agents (such as technological possibilities, preferences etc.) is called the set of economic environments $(E \neq \varnothing)$. The set of desired outputs (often resource allocations) of the action of economic agents is denoted by $Z$. Additionally, it is assumed that each agent sends messages available for communication, necessary for recognizing and defining his outputs.

Definition 3.1. The triple

$$
\Gamma=(M, \mu, h),
$$

where

- the set $M \neq \varnothing$, called the set of messages, contains the messages available to communications within agents, 
- the correspondence

$$
\mu: E \rightarrow M,
$$

called the message correspondence, associates with each element of environment $e \in E$, the set of messages $\mu(e) \subset M$

- the function

$$
h: M \rightarrow Z,
$$

called the outcome function, to every message $m \in M$ assigns the outcome $z \in Z$, is called the economic mechanism.

A function (correspondence)

$$
F: E \rightarrow Z,
$$

which to every environment $e \in E$ assigns the desirable or acceptable output (outputs) $z \in Z$, is called the goal function (correspondence).

Definition 3.2 (see (Hurwicz, Reiter 2006)). It is said that mechanism $\Gamma$ realizes the goal function if

$$
\forall e \in E \quad h(\mu(e))=F(e) .
$$

The person or institution coordinating agents' economic activity, who wants to get the set of outputs $Z$, later called the designer, has to recognize the set of messages $M$ and the message correspondence $\mu$. Finally, he has to define the outcome function $h$ realizing the goal function (or correspondence) $F$. The definition of outcome function is equivalent to the determination of organizing the economic activity in a given situation. If the designer finds more than one mechanism realizing the same outputs, he should choose the most appropriate mechanism, relative to an established criterion. This requires formulating criteria permitting to describe different economic systems.

If it is possible to make precise the set of environments $E^{a}(a \in\{1, \ldots, r\})$ of every agent $a$, then the set of environments $E$ can be defined as a Cartesian product

$$
E^{1} \times \ldots \times E^{r}
$$

where $e^{a}$ - the list of characteristics of agent $a$, is the $a-t h$ coordinate of the environment $e=\left(e^{1}, \ldots, e^{r}\right) \in E$. Analogously, the message correspondence of every agent is analyzed. Let 


$$
\mu^{a}: E^{a} \rightarrow M
$$

be the message correspondence of agent $a$. It associates with each element of environment $e^{a} \in E^{a}$, the set of messages $\mu^{a}\left(e^{a}\right) \subset M$. Additionally, a privacy preserving mechanism is defined:

Definition 3.3. The mechanism $\Gamma$ is the privacy preserving mechanism if for every $e=\left(e^{1}, \ldots, e^{r}\right) \in E$,

$$
\mu(e)=\bigcap_{a \in A} \mu^{a}\left(e^{a}\right) .
$$

If $\Gamma$ is a privacy preserving mechanism then correspondence $\mu^{a}$ (see (3.6)), depends only on the characteristics of agent $a$. This means that every agent knows only his own environment and, on the basis of the above, he sends his messages.

\section{The economy with a reduced consumption sphere}

Let $V \subset \mathbb{R}^{\ell}$ be a linear subspace of dimension $\ell-k, k \in\{1, \ldots, \ell-1\}$. Then $V$ is of the form

$$
V=\bigcap_{s=1}^{k} \operatorname{ker} \tilde{g}^{s}
$$

where

$$
\tilde{g}^{s}: \mathbb{R}^{\ell} \ni x \rightarrow x_{1} g_{1}^{s}+\ldots+x_{\ell} g_{\ell}^{s} \in \mathbb{R}
$$

are, for every $s \in\{1,2, \ldots, k\}$, the linear and continuous functions and vectors $g^{1}, \ldots, g^{k} \in \mathbb{R}^{\ell} \quad\left(g^{s}=\left(g_{1}^{s}, \ldots, g_{\ell}^{s}\right), s \in\{1,2, \ldots, k\}\right)$ are linearly independent. On the other hand, if a subset $V \subset \mathbb{R}^{\ell}$ is defined by (4.1) with functions satisfying (4.2), then $V$ is the linear subspace, of dimension $\ell-k$, of space $\mathbb{R}^{\ell}$.

Let $E_{p}=(P, C, \theta, \omega)$ be a Debreu economy. Assume that there exists a proper subspace $V$ of the commodity - price space $\mathbb{R}^{\ell}$ such that

$$
\forall i \in I \quad X^{i} \subset V
$$

(see (Lipieta 2010)). 
Definition 4.1. The private ownership Debreu economy $E_{p}=(P, C, \theta, \omega)$ in which condition (4.3) is satisfied for some subspace $V$ of commodity-price space $\mathbb{R}^{\ell}$ will be called the economy $E_{p}(V)$ with reduced consumption sphere.

Let us remember that if the quantities of some commodities are proportional (or at least approximately proportional; see (Lipieta 2010)) in all consumers' plans then condition (4.3) is satisfied. Notice also, that if all consumers are not interested in the consumption of at least one of the commodities offered by producers, then condition (4.3) is also satisfied. If the consumers do not want to consume a commodity $k_{0} \in\{1, \ldots, \ell\}$ then the coordinate $k_{0}$ is equal to 0 in every plan $x^{i} \in X^{i}$. Hence, for every $i \in I$,

$$
X^{i} \subset \operatorname{ker} \tilde{g} \stackrel{\text { def }}{=}\left\{x \in \mathbb{R}^{\ell}: \tilde{g}(x)=0\right\},
$$

where $\tilde{g}$ is of the form (4.2) and vector $g$ satisfies

$$
g=\left\{\begin{array}{lll}
1 & \text { for } & k=k_{0} \\
0 & \text { for } & k \neq k_{0}
\end{array} .\right.
$$

The set ker $\tilde{g}$ is the linear subspace of $\mathbb{R}^{\ell}$ of dimension $\ell-1$.

Similarly, we say that:

Definition 4.2. The private ownership Debreu economy $E_{p}=(P, C, \theta, \omega)$ in which condition

$$
\forall j \in J Y^{i} \subset V
$$

is satisfied for some subspace $V$ of commodity - price space $\mathbb{R}^{\ell}$ will be called the economy with reduced production sphere.

The private ownership Debreu economy satisfying condition (4.4) will also be denoted by $E_{p}(V)$. If there is a subspace $V$ of $\mathbb{R}^{\ell}$ such that conditions (4.3) and (4.4) are both satisfied in economy $E_{p}$, then this economy will be called the economy reduced to the subspace $V$. 


\section{Designing a mechanism in the private ownership economy with a reduced consumption sphere}

Let $E_{p}(V)$ be an economy with a reduced consumption sphere (see definitions 2.3, 4.1 and 4.2). This means (see (4.3)) that all the consumption plans are contained in the subspace $V$ of commodity-price space $\mathbb{R}^{\ell}$. Remember that in economy $E_{p}(V)$ conditions (2.1)-(2.4) are satisfied. Producers, observing the consumers' activity on the market, decide to change their production plans to satisfy the dependency between the quantities of commodities seen in the consumers' plans. The mechanism of the change in the production sphere while keeping the producers' maximal profits and equilibrium, if it existed, will be presented. However, now we present some results which will be of use later. The following theorem is the immediate consequence of theorem 4.2 by (Lipieta 2010):

Theorem 5.1. There exists a continuous and linear projection $Q: \mathbb{R}^{\ell} \rightarrow V$ such that for every $j \in J$ and $y^{j^{*}} \in \eta^{j}(p)$, vector $Q\left(y^{j^{*}}\right)$ maximizes (at price $p$ ) the profit of producer $j$ on the modified production set

$$
Q\left(Y^{j}\right)=\left\{Q\left(y^{j}\right) \in \mathbb{R}^{\ell}: y^{j} \in Y^{j}\right\} .
$$

Moreover,

$$
p \circ y^{j^{*}}=p \circ Q\left(y^{j^{*}}\right)
$$

for every $j \in J$ and $y^{j^{*}} \in \eta^{j}(p)$.

Remark 5.2. Let $Q: \mathbb{R}^{\ell} \rightarrow V$ be the projection by theorem 5.1. Then $Q$ is of the form

$$
Q(x)=x-\sum_{s=1}^{k} \tilde{g}^{s}(x) \cdot q^{s}
$$

where $\tilde{g}^{s}$ (see (Cheney 1966)), satisfies conditions (4.1)-(4.2) and vectors $q^{1}, \ldots, q^{k} \in \mathbb{R}^{\ell}$ are the solution of the system of equations:

$$
\left\{\begin{array}{l}
\tilde{g}^{s}(x)=\delta^{s r} \\
p \circ x=\delta
\end{array} s, r \in\{1, \ldots, k\}\right.
$$

if $p \notin V^{T} \stackrel{\text { def }}{=}\left\{x \in \mathbb{R}^{\ell} \mid \forall v \in V: p \circ v=0\right\}$, 
and the system of equations:

$$
\tilde{g}^{s}(x)=\delta^{s r} \quad s, r \in\{1, \ldots, k\}
$$

if $p \in V^{T}$. If every producer $j \in J$ changes his feasible production plans to the set $Q\left(Y^{j}\right)$ defined in condition (5.1), then the consumers' budget sets and optimal plans remain unchanged. Additionally, if

$$
\sum_{i=1}^{m} x^{i^{*}}-y=\omega \quad \text { then } \quad \sum_{i=1}^{m} x^{i^{*}}-Q(y)=\omega
$$

(see (4.1)-(4.2)) for every $y \in \mathbb{R}^{\ell}$.

We will analyze the theoretical example where the possible modification of production sphere in economy $E_{p}(V)$, according to the rules by theorem 5.1, will be presented. As a result we will get the economy reduced to subspace $V$.

Example 5.3. Let $E_{p}$ be a private ownership economy where two producers and only one consumer operate. Hence $J=\{1,2\}, I=\{1\}$ and by $(2.5), \theta(1,1)=1, \theta(1,2)=1$. Suppose that the producers' production sets are of the form

$$
\begin{gathered}
Y^{1}=-\mathbb{R}_{+}^{3}+\{(1,0,0)\}, \\
Y^{2}=\left\{\left(y_{1}, y_{2}, y_{3}\right) \in \mathbb{R}^{3}: y_{1}+4 y_{2}+2 y_{3} \leq 1 \wedge y_{1} \leq 1 \wedge y_{2} \leq 0\right\},
\end{gathered}
$$

where $\mathbb{R}_{+}=[0, \infty)$. Let us assume that the consumer's set is defined by

$$
\begin{gathered}
X=X^{1}=\left\{\left(x_{1}, x_{2}, x_{3}\right) \in \mathbb{R}_{+}^{3}: x_{2}=\frac{1}{10} x_{1} \wedge x_{3}=\frac{1}{2} x_{1}\right\}, \\
u^{1}\left(x_{1}, x_{2}, x_{3}\right)=x_{1}+x_{2}+x_{3} .
\end{gathered}
$$

Moreover, total endowment $\omega=(10,1,5)$ and price vector $p=(1,4,2) \in \mathbb{R}^{3}$ are given.

It is clear (by (5.7)) that in economy $E_{p}$ there are two couples of complementary commodities: the first and the second one, as well as the first and the third one. So the Debreu economy $E_{p}$ is the economy with a reduced consumption sphere, where subspace

$$
V=\left\{\left(v_{1}, v_{2}, v_{3}\right) \in \mathbb{R}^{3}: v_{2}=\frac{1}{10} v_{1} \wedge v_{3}=\frac{1}{2} v_{1}\right\}
$$


of the space commodities and prices $\mathbb{R}^{3}$ contains consumer's set $X(X \subset V)$. So $E_{p}=E_{p}(V)$.

By performing some calculations we get

$$
\begin{gathered}
y^{1^{*}}=(1,0,0), \\
y^{2^{*}}=\left(y_{1}, y_{2}, \frac{1}{2}\left(1-y_{1}-4 y_{2}\right)\right) \text { for } y_{1} \leq 1 \text { and } y_{2} \leq 0 .
\end{gathered}
$$

(see definition 2.1). Consequently, $\pi^{1}(p)=\pi^{2}(p)=1$. Moreover, by (2.6)

$$
w^{1}=26, x^{1^{*}}=\left(\frac{65}{6}, \frac{65}{60}, \frac{65}{12}\right) \text {. }
$$

To have condition (2.10) satisfied, the following should be fulfilled: $y_{1}^{2^{*}}=-\frac{1}{6}, y_{2}^{2^{*}}=\frac{5}{60}$. But plan $\left(-\frac{1}{6}, \frac{5}{60}, \frac{5}{6}\right)$ is not feasible for the second producer. As a result, there is no state of Walras equilibrium in economy $E_{p}(V)$. In this situation, the producers in economy $E_{p}(V)$ make the decision of changing their production activity according to condition (5.1).

By definition of subspace $V$ (condition (5.8)),

$$
\tilde{g}^{1}\left(x_{1}, x_{2}, x_{3}\right)=x_{2}-\frac{1}{10} x_{1}, \tilde{g}^{2}\left(x_{1}, x_{2}, x_{3}\right)=x_{3}-\frac{1}{2} x_{1}
$$

and consequently by (5.3),

$$
Q\left(x_{1}, x_{2}, x_{3}\right)=\left(x_{1}+4 x_{2}+2 x_{3}\right) \cdot\left(\frac{10}{24}, \frac{1}{24}, \frac{5}{24}\right) .
$$

Hence,

$$
\hat{Y}^{1}=\hat{Y}^{2}=\left\{v \cdot\left(1, \frac{1}{10}, \frac{1}{2}\right) \in \mathbb{R}^{3}: v \leq 1\right\}
$$

and $\hat{X}^{1}=X^{1}, \hat{u}^{1}=u^{1}, \hat{\omega}^{1}=\omega^{1}$.

All these calculations lead us to economy (see (Lipieta 2010)) $\hat{E}_{p}(V)$ reduced to the subspace $V$, where producers adjusted to the given relationship between quantities of commodities in their production plans. Now it is easy to calculate that

$$
\hat{y}^{1^{*}}=\hat{y}^{2^{*}}=\left(\frac{5}{24}, \frac{1}{24}, \frac{5}{24}\right), \hat{x}^{1^{*}}=x^{1}
$$

and the sequence 


$$
\left(\frac{65}{6}, \frac{65}{60}, \frac{65}{12}\right),\left(\frac{5}{24}, \frac{1}{24}, \frac{5}{24}\right),\left(\frac{5}{24}, \frac{1}{24}, \frac{5}{24}\right),(1,4,2)
$$

is the state of the Walras equilibrium in economy $\hat{E}_{p}(V)$.

Now, some examples of the designing economic mechanisms are presented. Firstly we should define the set of environments $E$. The set of environments in the economy $E_{p}(V)$ with reduced consumption sphere is defined on the basis of the characteristics of agents operating in this economy,

- $e^{j}=\left(Y^{j}\right) \in E^{j} \stackrel{\text { def }}{=} P\left(\mathbb{R}^{\ell}\right)$, for every producer $j \in J$,

- $e^{i}=\left(X^{i}, \omega^{i}, \preceq^{i}, \theta(i, \cdot)\right) \in E^{i} \stackrel{\text { def }}{=} P\left(\mathbb{R}^{\ell}\right) \times \mathbb{R}^{\ell} \times \Xi \times \mathcal{F}$, where

$$
\mathcal{F}=\{f: I \times J \rightarrow[0,1]\} \text {, for every consumer } i \in I \text {. }
$$

Hence (see (3.5)), the set of environments is of the form

$$
E \stackrel{\text { def }}{=} E^{i_{1}} \times \ldots \times E^{i_{m}} \times E^{j_{1}} \times \ldots \times E^{j_{n}} .
$$

Let us notice, that theorem 5.1 shows that every producer $j$ can modify his productive activity to plans contained in subspace $V$, all the time having the possibility of maximizing the profit. Denoting, for every producer $j$,

- $\hat{Y}^{j}$ the modified production set, contained in the subspace $V$,

- $\hat{\eta}^{j}(p) \stackrel{\text { def }}{=}\left\{\hat{y}^{j^{*} \in \hat{Y}^{j}}: p \circ \hat{y}^{j^{*}}=\max \left\{p \circ \hat{y}^{j}: \hat{y}^{j} \in \hat{Y}^{j}\right\}\right\}$ the set of his optimal plans, we define the set of outputs

$$
\begin{gathered}
Z \stackrel{\text { def }}{=}\left\{\left(x^{1^{*}}, \ldots, x^{m^{*}}, \hat{y}^{1^{*}}, \ldots, \hat{y}^{n^{*}}\right) \in(V)^{m+n}:\right. \\
\left.\exists p \in T \mid \forall j \in J \hat{y}^{j^{*}} \in \hat{\eta}^{j}(p) \wedge \forall i \in x^{i^{*}} \in \varphi^{i}(p), \sum_{i=1}^{m} x^{i^{*}}-\sum_{j=1}^{n} \hat{y}^{j^{*}}=\omega\right\},
\end{gathered}
$$

where

$$
T=\left\{p \in \mathbb{R}^{\ell}: \forall j \in J \eta^{j}(p) \neq \varnothing \wedge \forall i \in I \varphi^{i}\left(p, w^{i}\right) \neq \varnothing\right\} .
$$

If $Z \neq \varnothing$, then we define the goal correspondence $F: E \rightarrow Z$ which to the set of economic agents represented by environment $e \in E$ by (5.10), assigns the sequence of agents' optimal plans satisfying condition (2.8), which gives the equilibrium in the economy. 
Theorem 5.4. Assume that there is a state of equilibrium in economy $E_{p}(V)$ at given price vector $p \in \mathbb{R}^{\ell}$. Then there is a privacy preserving mechanism $\Gamma=(M, \mu, h)$ realizing the goal correspondence $F: E \rightarrow Z$, where set $E$ is of the form (5.10) and nonempty set $Z$ of the form (5.11).

Proof. By theorem 5.1 and remark $5.2, Z \neq \varnothing$. The set

$$
\begin{gathered}
M \stackrel{\text { def }}{=}\left\{m=\left(p, x^{1}, \ldots, x^{m}, y^{1}, \ldots, y^{n}\right) \in \mathbb{R}^{\ell} \times X^{1} \times \ldots \times X^{m} \times(V)^{n}:\right. \\
\left.\sum_{i=1}^{m} x^{i}-\sum_{j=1}^{n} y^{j}=\omega\right\},
\end{gathered}
$$

by assumption of the existence of the state of equilibrium in economy $E_{p}(V)$, is the nonempty set of messages. Define

- message correspondence of every producer $j, \mu^{j}: E^{j} \rightarrow M$ :

$$
\mu^{j}\left(e^{j}\right)=\mu^{j}\left(Y^{j}\right)=\left\{m \in M: p \in T \wedge y^{j^{*}} \in \eta^{j}(p)\right\},
$$

- the message correspondence of every consumer $i, \mu^{i}: E^{i} \rightarrow M$ :

$$
\mu^{i}\left(e^{i}\right)=\mu^{i}\left(X^{i}, \omega^{i}, \preceq^{i}, \theta(i, \cdot)\right)=\left\{m \in M: p \in T \wedge x^{i^{*}} \in \varphi^{i}(p)\right\},
$$

- message correspondence $\mu: E \rightarrow M$ :

$$
\begin{gathered}
\mu(e)=\left(e^{1}, \ldots, e^{m+n}\right)=\bigcap_{a=1}^{\operatorname{def}} \mu^{a+m}\left(e^{a}\right)=\left\{m=\left(p, x^{1^{*}}, \ldots, x^{m^{*}}, y^{1^{*}}, \ldots, y^{n^{*}}\right) \in M:\right. \\
\left.p \in T, \forall j \in J y^{j^{*}} \in \eta^{j}(p), \forall i \in I x^{i^{*}} \in \varphi^{i}(p)\right\} .
\end{gathered}
$$

Defining the outcome function $h: M \rightarrow Z$, of the form

$$
h\left(p, x^{1^{*}}, \ldots, x^{m^{*}}, y^{1^{*}}, \ldots, y^{n^{*}}\right) \stackrel{\operatorname{def}}{=}\left(x^{1^{*}}, \ldots, x^{m^{*}}, Q\left(y^{1^{*}}\right), \ldots, Q\left(y^{n^{*}}\right)\right),
$$

where $Q$ is the projection by the thesis of theorem 5.1, we get that the privacy preserving (by (5.14); see def. 3.3) mechanism $\Gamma=(M, \mu, h)$ satisfies the thesis of the theorem.

Remember that in the mechanism defined in theorem 5.4, every agent sends his messages on the basis of the knowledge only of his own environment (see def. 3.3). Additionally, if the system of equalities, appropriate to (5.4) or (5.5), has infinitely many solutions, then there are infinitely many 
mechanisms defined in theorem 5.4 satisfying the goal correspondence $F$ with the set of environments (5.10) and the set of outputs (5.11). In some particular cases, it is possible to define the similar economic mechanisms without the assumption of the existence of equilibrium in economy $E_{p}(V)$. For instance, the following is true:

Theorem 5.5. Let $E_{p}(V)$ be an economy with a reduced consumption sphere and $Q$ be the projection of the form (5.3), satisfying

$$
Q\left(x^{*}-y^{*}-\omega\right)=0 \text {. }
$$

Then there is a privacy preserving mechanism realizing the goal function $F: E \rightarrow Z$, where $E$ is of the form (5.10) and $Z$ of the form (5.11).

Proof. Let $\hat{E}_{p}(V)$ be the modification of economy $E_{p}(V)$ in which all the production sets $Y^{j}$ are replaced by sets $Q\left(Y^{j}\right)$. By (5.15), (4.3) and (5.3),

$$
x^{*}-Q\left(y^{*}\right)-\omega=0,
$$

which means, after taking into account the results of theorem 5.1, that there is the state of equilibrium in economy $\hat{E}_{p}(V)$. Hence the sets: $M$ by (5.13) and $Z$ by (5.15) are nonempty. The rest of the proof goes on the same as the proof of theorem 5.4

Finally, we prove the following:

Theorem 5.6. The economy $E_{p}(V)$, reduced to the subspace $V$, is the privacy preserving mechanism realizing the goal correspondence $F: E \rightarrow Z$, where the set of environments is of the form (5.10), and the set of outcomes

$$
Z \stackrel{\text { def }}{=}\left\{\left(p, X^{1}, \ldots, X^{m}, \hat{Y}^{1}, \ldots, \hat{Y}^{n}\right) \in \mathbb{R}^{\ell} \times(P(V))^{m+n}\right\} .
$$

Proof. Let

$$
M \stackrel{\text { def }}{=}\left\{m=\left(p, \hat{X}^{1}, \ldots, \hat{X}^{m}, \hat{Y}^{1}, \ldots, \hat{Y}^{n}\right) \in \mathbb{R}^{\ell} \times\left(P\left(\mathbb{R}^{\ell}\right)\right)^{m+n}\right\},
$$

be a (nonempty) set of messages. Let set $T$ be of the form (5.12) and $Q$ be a projection by the thesis of theorem 5.1 . Then

- message correspondence of producer $j, \mu^{j}: E^{j} \rightarrow M$ :

$$
u^{j}\left(e^{j}\right)=u^{j}\left(Y^{j}\right)=\left\{m \in M: p \in T \wedge \hat{Y}^{j}=Q\left(Y^{j}\right)\right\},
$$


- the message correspondence of consumer $i, \mu^{i}: E^{i} \rightarrow M$ :

$$
u^{i}\left(e^{i}\right)=\mu^{i}\left(X^{i}, \omega^{i}, \preceq^{i}, \theta(i, \cdot)=\left\{m \in M: p \in T \wedge \hat{X}^{i}=Q\left(X^{i}\right)\right\},\right.
$$

- message correspondence $\mu: E \rightarrow M$ :

$$
\begin{gathered}
\mu(e)=\mu\left(e^{1}, \ldots, e^{m+n}\right) \stackrel{\text { def }}{=} \bigcap_{a=1}^{n+m} \mu^{a}\left(e^{a}\right)= \\
\left\{m=\left(p, X^{1}, \ldots, X^{m}, Q\left(Y^{\left(1^{*}\right)}\right), \ldots, Q\left(Y^{n}\right) \in M: p \in T\right\} .\right.
\end{gathered}
$$

Defining the outcome function $h: M \rightarrow Z$, of the form

$$
h\left(p, X^{1}, \ldots, X^{m}, Y^{1}, \ldots, Y^{n}\right) \stackrel{\text { def }}{=}\left(p, X^{1}, \ldots, X^{m}, Y^{1}, \ldots, Y^{1}\right)
$$

we get that privacy preserving (by (5.17)) mechanism $\Gamma=(M, \mu, h)$ satisfies the thesis of the theorem.

Notice, analogously as above, that if the system of equalities appropriate to (5.4) or (5.5) has infinitely many solutions, then there are infinitely many mechanisms realizing the goal correspondence $F: E \rightarrow Z$, with the set of environments $E$ defined in (5.10) and the set of outputs $Z$ of the form (5.16). This means that there are infinitely many possibilities of changing the production sphere to the production plans contained in the subspace of the commodity - price space, designed by the dependency between the quantities of commodities in the consumers' plans. Moreover, the equilibrium will be not destroyed, if it exists in the initial economy with a reduced consumption sphere. The nonempty (by theory 5.1) set of outputs (5.16) form the economy reduced to the subspace (see (Lipieta 2010)).

\section{Conclusion}

The existence of the reduced consumption sphere in the Debreu private ownership economy enables to simplify the geometrical structure of this economy. The reduction of the dimension of the commodity - price space has positive effects for further theoretical analyses such as determining, in some cases, the economic mechanism as a result of which the equilibrium can be achieved or to provide the evolution of the production sphere with keeping the equilibrium. 


\section{References}

Arrow K.J., Intriligator M.D. (eds.) (1987). Handbook of Mathematical Economics. Vol. 3. Amsterdam. North-Holland.

Cheney E.W. (1966). Introduction to Approximation Theory. McGraw-Hill. New York.

Debreu G. (1959). Theory of value. New York. Wiley.

Hurwicz L., Reiter S. (2006). Designing Economic Mechanism. Cambridge University Press. New York.

Lipieta A. (2010). The Debreu private ownership economy with complementary commodities and prices. Economic Modelling 27. Pp. 22-27.

Malawski A. (2005). A dynamical system approach to the Arrow-Debreu theory of general equilibrium. The $9^{\text {th }}$ World Multi - Conference on Systemics, Cybernetics, and Informatics Proceedings. Vol. 7. July 10-13. Orlando. Florida. USA.

Werner J. (2010). Theory of mechanism design. Course Handouts. Warsaw. 